\title{
ASYMPTOTIC SOLUTIONS OF DIFFUSION MODELS FOR RISK RESERVES
}

\author{
S. SHAO
}

Received 22 August 2002

\begin{abstract}
We study a family of diffusion models for risk reserves which account for the investment income earned and for the inflation experienced on claim amounts. After we defined the process of the conditional probability of ruin over finite time and imposed the appropriate boundary conditions, classical results from the theory of diffusion processes turn the stochastic differential equation to a special class of initial and boundary value problems defined by a linear diffusion equation. Armed with asymptotic analysis and perturbation theory, we obtain the asymptotic solutions of the diffusion models (possibly degenerate) governing the conditional probability of ruin over a finite time in terms of interest rate.
\end{abstract}

2000 Mathematics Subject Classification: 35K20, 35B25, 41A60, 91B30.

1. Introduction. The diffusion-type modeling for risk reserves is one of the most popular methods for the valuing of the investment income earned and for the inflation experienced on claim amounts in studying insurance models. The basic assumption is that the risk reserve is modeled as one-dimensional diffusion process. In a continuous time aspect of the system under study, diffusion process is an arbitrary strong Markov process with continuous sample paths, or even more specifically, diffusion process is given as a strong solution of a stochastic differential equation driven by the underlying Brownian motion. This approach, which uses an appropriate diffusion process to approximate the actual claims process, has been examined by many authors during the past, including Garrido [5, 6], Harrison [8], Iglehart [9], Willmot and Yang [13], Moller [10], Young and Zariphopoulou [14], and many others during the late 80 s to 2000. Garrido [5] studied a family of diffusion models for risk reserves, he obtained an explicit solution of the stochastic differential equation for the distribution of the time to ruin for the linear case. Willmot and Yang studied a modified diffusion models by allowing the variance to depend on the size of the reserve, which made the model more realistic. They provided some solutions for the partial differential equations obtained from the stochastic differential equations in some special cases. Young and Zariphopoulou studied the distorted probabilities for the nondegenerate diffusion processes. In this paper, we focus on the solutions of diffusion models with possibly degenerate diffusion, that is, we allow the case of vanishing viscosity. Our first effort is to 
impose the appropriate boundary condition for the diffusion models for risk reserves, then the classical results from the theory of diffusion processes and linear partial differential equations yield a special class of initial and boundary value problems defined by an unsteady linear diffusion equation. This is particularly important because although the study of partial differential equations in complete generality is a vast undertaking, almost all the partial differential equations encountered in financial applications belong to a much more manageable subset of the whole second-order linear parabolic equations [3]. By allowing the possibly dependencies of the diffusion process, our second focus is on providing the asymptotic solutions of the linear diffusion equations at the opened upper half of vertical axis

$$
\begin{gathered}
\frac{\partial \phi}{\partial t}+\left(\alpha+r \beta e^{A(t)}\right) \frac{\partial \phi}{\partial r}+\frac{1}{2} \sigma^{2} \frac{\partial^{2} \phi}{\partial r^{2}}=0, \\
\phi(t, r)=1, \quad r<0, \\
\phi(T, r)=0, \quad r>0,
\end{gathered}
$$

where $\phi \in C^{2}([0, T) \times \mathbb{R}), 0 \leq t \leq T<\infty, \alpha$ is a constant, the interest rate $\beta>0$, the diffusion coefficient $\sigma>0$, and $A(t)$ is a continuous function in the given domain. Although numerical solutions of boundary value problems of partial differential equations often use a variety of finite difference or finite element formulations of differential equations, it is also quite common to convert given problems into integral equations, from which boundary element methods can be applied to obtain numerical approximations. Moreover, we notice that the boundary condition (1.2) is defined on the left-half opened space $r<0$. The analytic solution of (1.1), (1.2), and (1.3) is unlikely to be obtained because of such initial and boundary conditions. Using asymptotic analysis and perturbation theory, we obtain the conditional probability of ruin $\phi(t, r)$ over a finite time by providing the asymptotic solutions of the diffusion equations in terms of interest rate $\beta$ and the volatility (diffusion coefficient) $\sigma$. Our aim is to understand the asymptotic behavior of the conditional probability of ruin $\phi(t, r)$ between time $t$ and $T$ with reserve $r$ at time $t$. Our results show that when the difference $\alpha$ of the aggregate rate $\pi(t, r)$ at which premiums are cashed at that time and the average aggregated claim rate $\mu(t)$ at time $t$ is greater than zero, there are boundary layer and parabolic corner layer behavior in the solutions of when volatility coefficient $\sigma$ is degenerated, the interest rate $\beta$ has little impact to the asymptotic solutions in this case whether it is small or not. For $\alpha \leq 0$, no boundary layer or corner layer occurs, the asymptotic solutions of (1.1), (1.2), and (1.3) can be obtained in terms of the interest rate and it is uniformly valid for the whole domain. We hope the explicit asymptotic solutions are useful to illustrate the general behavior of the solutions for more complicated case such as the multiple portfolios case.

In Section 2, we state some basic definitions and results from the classical theory of stochastic processes and derive a family of the linear diffusion 
equation with its initial and boundary conditions. Some useful results from asymptotic analysis are given before we close this section. In Section 3, we study the asymptotic behavior of solutions of (1.1), (1.2), and (1.3) under different behavior of $\sigma$ and $\alpha$. Some results are illustrated by the graphs of the asymptotic solutions at the end of the section. The final conclusion and remarks will be discussed in Section 4.

2. Preliminaries and boundary conditions of the parabolic equation. In this section, we start with a brief review from the fundamental results of the theory of stochastic calculus, then we impose appropriate boundary conditions to the partial differential equations obtained from the diffusion models. Some useful results from asymptotic analysis will be stated before we close the section. Most of the results can be found in [1, 2, 4, 5, 7, 11, 12, 13].

DEFinition 2.1. Let $R(t)$ be the risk reserve level at time $t$ and let $F_{t}=$ $\sigma\left\{W_{s}: 0 \leq s \leq t\right\}$ be the $\sigma$ field generated by a standard Brownian motion $W_{t}$. Then, the following definitions can be stated.

(a) The conditional probability $\phi(t, r)$ defined by

$$
\phi(t, r)=P\left(\inf _{t \leq s \leq T} R(s)<0 \mid R(t)=r\right)
$$

is called the probability of ruin between $t$ and $T$ given the reserve $r$ at time $t$.

We notice that ruin could happen, for instance, if the company pays out pension, or if it invests its reserve and gets a negative outcome of its investments. By definition (a), the probability of ruin is one when the reserve becomes negative, that is, $\phi(t, r)=1, r<0$, with $0 \leq t<T \leq \infty$ and the initial probability of ruin is zero at time $t=T$ when the reserve is positive. Therefore, we obtain condition (1.3).

(b) The time $\tau$ defined by

$$
\tau=\inf \{t \geq 0 \mid R(t)<0\}
$$

is the first time that the reserve level becomes negative and is called the time of ruin.

(c) A process $S$ is called a martingale with respect to a measure $P$ and a family of $\sigma$-field $F_{i}$ if

$$
E_{p}\left(S_{j} \mid F_{i}\right)=S_{i}, \quad \forall i \leq j
$$

THEOREM 2.2. Suppose that $g$ is a function of class $C^{2}\left(\mathbb{R}^{k}, \mathbb{R}\right)$ and the processes $X^{i}$ is in the class of all real-valued continuous semimartingales on the probability space for $i=1,2, \ldots, k$, then the following form of Ito's formula 
is valid:

$$
\begin{aligned}
g(X)-g\left(X_{0}\right)= & \sum_{i=1}^{k} \int_{0}^{t} \frac{\partial g}{\partial x_{i}}\left(X_{u}\right) d X_{u}^{i} \\
& +\frac{1}{2} \sum_{i, j=1}^{k} \int_{0}^{t} \frac{\partial^{2} g}{\partial x_{i} x_{j}}\left(X_{u}\right) d\left(\left\langle X^{i}, X^{j}\right\rangle_{u}\right) .
\end{aligned}
$$

THEOREM 2.3. If $\phi(t \wedge \tau, R(t \wedge \tau))$ is an $F_{t \wedge \tau}$ martingale, that is,

$$
\phi(t \wedge \tau, R(t \wedge \tau))=E\left(I(\tau<T) \mid F_{t \wedge \tau}\right),
$$

where $t \wedge \tau=\min \{t, \tau\}$, then the conditional probability of ruin $\phi(t, r)$ satisfies the parabolic equation

$$
\frac{\partial \phi}{\partial t}+\gamma(t, r) \frac{\partial \phi}{\partial r}+\frac{1}{2} \sigma^{2}(t, r) \frac{\partial^{2} \phi}{\partial r^{2}}=0
$$

where $\gamma(t, r)=[\pi(t, r)+\beta(t) r-\mu(t)]$ is defined as in (2.7).

Now, consider the risk reserves accumulated for a particular line of business. The risk reserve level $R(t)$ satisfies the following stochastic differential equation:

$$
\begin{aligned}
d R(t) & =[\pi(t, R(t))+\beta(t) R(t)-\mu(t)] d t+\sigma(t, R(t)) d W_{t}, \\
R(0) & =r_{0} \geq 0
\end{aligned}
$$

Here, $\pi(t, R(t))$ is the aggregate rate at which premiums are cashed at time $t$, $\mu(t)$ is the average aggregated claim rate at time $t, \beta(t)$ is assumed the known force of interest at that time, and $W_{t}$ is a standard Brownian motion. Since the coefficients of the stochastic differential equation (2.7) depend only on the history through $R(t)$, therefore, the reserve process $R(t)$ is a Markov process. The strong Markovian property of $R(t)$ implies that $\phi(t \wedge \tau, R(t \wedge \tau))$ is an $F_{t \wedge \tau}$ martingale. Thus, $\phi(t \wedge \tau, R(t \wedge \tau))-\phi_{0}\left(0, r_{0}\right)$ is a zero initial-valued martingale, and it is square integrable. Apply Ito's formula in Theorem 2.2, then the following linear diffusion equation can be obtained by Theorem 2.3:

$$
\frac{\partial \phi}{\partial t}+[\pi(t, r)+\beta(t) r-\mu(t)] \frac{\partial \phi}{\partial r}+\frac{1}{2} \sigma^{2} \frac{\partial^{2} \phi}{\partial r^{2}}=0
$$

Let $\pi(t, r)-\mu(t)=\alpha$ be a constant; $\sigma(t)=\sigma>0, \beta(t)=\beta e^{A(t)}$ with $\beta>0$, and $A(t)=\int_{0}^{t} \lambda(s) d s$, where $\lambda(t)$ acts as a functional force of inflation on claims and premiums. Then we obtain the linear diffusion equation (1.1). By the definition of $\phi(t, r)$ in (a) and the previous discussion, the conditional probability of ruin $\phi(t, r)$ satisfies the initial and boundary conditions (1.2) and (1.3) where $0 \leq t \leq T<\infty$. 
We notice that there is no boundary condition defined along $r=0$. We will discuss the asymptotic solutions of (1.1), (1.2), and (1.3) across the discontinuity $r=0$ in Section 3 .

Before closing this section, we introduce a useful lemma and a theorem in asymptotic analysis.

LEMMA 2.4. For $-\infty<x<\infty$,

$$
\begin{aligned}
& \frac{1}{\sqrt{\pi \epsilon}} \int_{-\infty}^{x} \exp \left(-\frac{\eta^{2}}{\epsilon}\right) d \eta=1-\frac{1}{2} \operatorname{erfc}\left(\frac{x}{\epsilon}\right) \\
& \frac{1}{\sqrt{\pi \epsilon}} \int_{x}^{\infty} \exp \left(-\frac{\eta^{2}}{\epsilon}\right) d \eta=\frac{1}{2} \operatorname{erfc}\left(\frac{x}{\epsilon}\right)
\end{aligned}
$$

for all $\epsilon>0$.

THEOREM 2.5. The integral $I_{a}(x, t)$ defined by

$$
I_{a}(x, t)=\sqrt{\frac{2}{\pi \sigma^{2}}} \int_{-\infty}^{(x+\alpha t) / 2 \sqrt{t}} \Omega(\eta ; x, t) \exp \left(-\frac{\eta^{2}}{\sigma^{2} / 2}\right) d \eta
$$

can be expanded as

$$
I_{a}(x, t)=I_{a}^{(0)}(t, x)+\sigma I_{a}^{(1)}(x, t)+\sigma^{2} I_{a}^{(2)}(x, t),
$$

where

$$
\begin{aligned}
& I_{a}^{(0)}(x, t)=\Omega(0 ; x, t)-\frac{1}{2} \Omega\left(\frac{x+\alpha t}{2 \sqrt{t}} ; x, t\right) \operatorname{erfc}\left(\frac{x+\alpha t}{\sigma \sqrt{2 t}}\right), \\
& I_{a}^{(1)}(x, t)=\frac{\sqrt{t}}{x+\alpha t}\left(\Omega(0 ; x, t)-\Omega\left(\frac{x+\alpha t}{2 \sqrt{t}} ; x, t\right)\right) \operatorname{ierfc}\left(\frac{x+\alpha t}{\sigma \sqrt{2 t}}\right), \\
& I_{a}^{(2)}(x, t)=\frac{1}{\sigma \sqrt{2 \pi}} \int_{-\infty}^{(x+\alpha t) / 2 \sqrt{t}} \Omega^{(2)}(\eta ; x, t) \exp \left(-\frac{\eta^{2}}{\sigma^{2} / 2}\right) d \eta,
\end{aligned}
$$

with

$$
\Omega^{(2)}(\eta ; x, t)=\frac{d}{d \eta}\left(\frac{1}{\eta}\{\Omega(\eta ; x, t)-\Omega(0 ; x, t)\}\right) .
$$

The integral $I_{b}(x, t)$ defined by

$$
I_{b}(x, t)=\sqrt{\frac{2}{\pi \sigma^{2}}} \int_{(x-\alpha t) / 2 \sqrt{t}}^{\infty} \Omega(\eta ; x, t) \exp \left(-\frac{\eta^{2}}{\sigma^{2} / 2}\right) d \eta
$$

can be expanded as

$$
I_{b}(x, t)=I_{b}^{(0)}(x, t)+\sigma I_{b}^{(1)}(x, t)+\sigma^{2} I_{b}^{(2)}(x, t),
$$


where $\alpha<0,0<\sigma \ll 1$,

$$
\begin{aligned}
& I_{b}^{(0)}(x, t)=\frac{1}{2} \Omega\left(\frac{x-\alpha t}{2 \sqrt{t}} ; x, t\right) \operatorname{erfc}\left(\frac{x-\alpha t}{\sigma \sqrt{2 t}}\right), \\
& I_{b}^{(1)}(x, t)=\frac{\sqrt{t}}{x-\alpha t}\left(\Omega\left(\frac{x-\alpha t}{2 \sqrt{t}} ; x, t\right)-\Omega(0 ; x, t)\right) \operatorname{ierfc}\left(\frac{x-\alpha t}{\sigma \sqrt{2 t}}\right), \\
& I_{b}^{(2)}(x, t)=\frac{1}{\sigma \sqrt{2 \pi}} \int_{(x-\alpha t) / 2 \sqrt{t}}^{\infty} \Omega^{(2)}(\eta ; x, t) \exp \left(-\frac{\eta^{2}}{\sigma^{2} / 2}\right) d \eta,
\end{aligned}
$$

where

$$
\begin{aligned}
\operatorname{erfc}(x) & =\frac{2}{\sqrt{\pi}} \int_{x}^{\infty} e^{-s^{2}} d s \sim \frac{1}{x \sqrt{\pi}} \exp \left(-x^{2}\right), \text { as } x \rightarrow \infty \\
\operatorname{ierfc}(x) & =\frac{1}{\sqrt{\pi}} \exp \left(-x^{2}\right)-x \operatorname{erfc}(x) \sim \frac{1}{2 x^{2} \sqrt{\pi}} \exp \left(-x^{2}\right), \quad \text { as } x \rightarrow \infty .
\end{aligned}
$$

Moreover, if $\Omega(\eta ; x, t)$ is a polynomial of degree $n$ in $\eta$, then $\Omega^{(2)}(\eta ; x, t)$ is a polynomial of degree $n-2$ in $\eta$.

These results can be found in [12] and will be used in our later sections.

3. Asymptotic solutions of the diffusion equations. Consider the linear parabolic differential equation

$$
\frac{\partial \phi}{\partial t}+\left(\alpha+r \beta e^{A(t)}\right) \frac{\partial \phi}{\partial r}+\frac{1}{2} \sigma^{2} \frac{\partial^{2} \phi}{\partial r^{2}}=0
$$

subject to initial and boundary conditions

$$
\begin{array}{cl}
\phi(t, r)=1, & r<0, \\
\phi(T, r)=0, & r>0,
\end{array}
$$

where $0 \leq t \leq T \leq \infty$. Letting $\tau=T-t$, and making change of variables in (3.1) and (3.2), we have

$$
\begin{gathered}
\frac{\partial \phi}{\partial \tau}=\left(\alpha+r \beta e^{A(\tau)}\right) \frac{\partial \phi}{\partial r}+\frac{1}{2} \sigma^{2} \frac{\partial^{2} \phi}{\partial r^{2}}, \\
\phi(\tau, r)=1, \quad r<0, \\
\phi(0, r)=0, \quad r>0 .
\end{gathered}
$$

Since the boundary condition (3.4) is defined for $r<0$, in order to obtain a smooth transition for the asymptotic solutions of (3.3), (3.4), and (3.5) across the discontinuity boundary $r=0$, we use the boundary layer theory to determine the asymptotic behavior of solution of (3.2), (3.3), (3.4), and (3.5) in the neighborhood of $r=0$. Define the stretched variable $\xi$ along $r=0$ by

$$
\xi=\frac{r}{\epsilon^{k}}
$$


insert (3.6) into (3.3), and evaluate the coefficient of $\partial \phi / \partial r$ at $r=0$. This gives, with $\psi=\psi(\tau, \xi)$,

$$
\frac{\partial \psi}{\partial \tau}=\epsilon^{-k} \alpha \frac{\partial \psi}{\partial \xi}+\frac{1}{2} \sigma^{2} \epsilon^{-2 k} \frac{\partial^{2} \psi}{\partial \xi^{2}}
$$

We now divide our discussions into two Cases (I) $\alpha>0$ and (II) $\alpha \leq 0$.

CASE (I) $(\alpha>0)$. If $\sigma$ is small, without loss of generality, let $O(\sigma) \sim O(\epsilon)$, then we choose $k=2$ in (3.7) to balance the leading term, this gives the boundary layer equation

$$
2 \epsilon^{2} \frac{\partial \psi}{\partial \tau}=2 \alpha \frac{\partial \psi}{\partial \xi}+\frac{\partial^{2} \psi}{\partial \xi^{2}}
$$

which replaces (3.3). With $\psi$ expended in powers of $\epsilon$ and with $\psi_{0}$ as its leading term, we find that

$$
\psi_{0}(\tau, \xi)=a(\tau)+b(\tau) e^{-2 \alpha \xi}
$$

where $a$ and $b$ are arbitrary functions to be determined using the matching condition and the boundary condition so that a boundary layer of width $O\left(\sigma^{2}\right)$ can be located near $r=0$. On the other hand, leading term of the outer solution of (3.3) satisfies the reduced equation

$$
\frac{\partial \phi_{0}}{\partial \tau}=0, \quad 0=\left(\alpha+\beta e^{A(\tau)}\right) \frac{\partial \phi_{0}}{\partial r}, \quad r>0,
$$

which implies

$$
\phi_{0}(\tau, r)=0, \quad r>0
$$

Using the method of matching with the matching condition and the boundary conditions

$$
\begin{aligned}
\lim _{\xi \rightarrow 0} \psi(\tau, \xi) & =\lim _{r \rightarrow 0} \phi(\tau, r)-\phi_{0}(\tau, 0)=1, \\
\lim _{\xi \rightarrow \infty} \psi(\tau, \xi) & =0,
\end{aligned}
$$

we have the boundary layer solution of $O\left(\sigma^{2}\right)$,

$$
\psi_{0}(\tau, \xi)=e^{-2 \alpha \xi}, \quad \xi>0 .
$$

To determine if there is a corner layer near origin $(0,0)$, we introduce a stretch variable $\varsigma$ along $t=0$, defined by

$$
\varsigma=\frac{\tau}{\epsilon^{2}}
$$


and the corner layer $\varphi(\varsigma, \xi)$ to (3.8), we obtain a corner layer equation which is a linear parabolic partial differential equation of the form

$$
\frac{\partial \varphi}{\partial \varsigma}=\alpha \frac{\partial \varphi}{\partial \xi}+\frac{1}{2} \frac{\partial^{2} \varphi}{\partial \xi^{2}}
$$

for $0<\xi<\infty, 0<\varsigma<\infty$ subject to the initial and boundary conditions

$$
\begin{gathered}
\varphi(0, \xi)=-\psi_{0}(0, \xi)=-e^{2 \alpha \xi}, \quad \xi>0, \\
\varphi(\varsigma, 0)=0, \quad \varsigma>0, \\
\lim _{\xi \rightarrow \infty} \varphi(\varsigma, \xi)=0, \quad \varsigma>0 .
\end{gathered}
$$

Solving (3.15) and (3.16), we obtain the parabolic corner layer solution of width $O\left(\sigma^{2}\right)$,

$$
\varphi(\varsigma, \xi)=-e^{-2 \alpha \xi-\alpha^{2} \varsigma / 2} \operatorname{erf}\left(\frac{\xi}{\sqrt{2 \zeta}}\right)
$$

where the error function erf is defined by

$$
\operatorname{erf}(x)=\frac{2}{\sqrt{\pi}} \int_{0}^{x} e^{-s^{2}} d s
$$

If, in addition, $\beta$ is small, assume that $O\left(\beta^{s}\right) \sim O(\sigma)$ for some $s>0$, we have the boundary layer and parabolic corner layer as

$$
\begin{gathered}
\psi_{0}(\tau, \xi)=e^{-2 \alpha \xi}, \quad \xi=\frac{r}{\beta^{2 s}}, \\
\varphi(\varsigma, \xi)=-e^{-2 \alpha \xi-\alpha^{2} \varsigma / 2} \operatorname{erf}\left(\frac{\xi}{\sqrt{2 \varsigma}}\right), \quad \varsigma=\frac{\tau}{\beta^{2 s}} .
\end{gathered}
$$

Therefore, we have the following theorem.

THEOREM 3.1. If $\alpha>0$ and $O(\sigma)$ is a small positive parameter, the asymptotic solution, as $\sigma \rightarrow 0^{+}$, of (1.1), (1.2), and (1.3) has the following form:

$$
\begin{aligned}
\phi(t, r) \approx & e^{-2 \alpha r / \sigma^{2}} \\
& \times\left(1-e^{-\alpha^{2}(T-t) / 2 \sigma^{2}} \operatorname{erf}\left(\frac{r}{\sigma \sqrt{2(T-t)}}\right)\right), \quad r>0,0<t \leq T<\infty, \\
\phi(t, r)= & 1, \quad r<0 .
\end{aligned}
$$


If, in addition, $O\left(\beta^{s}\right) \sim O(\sigma)$ for some $s>0$, then the asymptotic solution, as $\beta \rightarrow 0^{+}$, of (1.1), (1.2), and (1.3) has the following form:

$$
\begin{aligned}
\phi(t, r) \approx & e^{-2 \alpha r / \beta^{2 s}} \\
& \times\left(1-e^{-\alpha^{2}(T-t) / 2 \beta^{2 s}} \operatorname{erf}\left(\frac{r}{\beta^{s} \sqrt{2(T-t)}}\right)\right), \quad r>0,0<t \leq T<\infty, \\
\phi(t, r)= & 1, \quad r<0 .
\end{aligned}
$$

REMARK 3.2. For a noise problem of (3.7), we replace $\sigma$ by the noise coefficient $\sigma \epsilon$ with $\epsilon \rightarrow 0^{+}$and we can obtain a similar result using the above techniques to obtain the asymptotic solutions of (3.1) and (3.2).

CASE (II) $(\alpha \leq 0)$. (i) For the case $O(\sigma) \sim O(\epsilon)$, no boundary layer occurs since the exponential term decreases as $\xi$ decreases in (3.9), but $\phi(\tau, r)=1$ for $r<0$.

(ii) When $\sigma$ is not a small parameter if we retain the stretching variables $\xi=r / \beta^{k}$ and substitute into (3.3) with $\psi=\psi(\tau, \xi)=\phi(\tau, r)$, the boundary layer equation becomes

$$
\beta^{2 k} \frac{\partial \psi}{\partial \tau}=\beta^{k} \alpha \frac{\partial \psi}{\partial \xi}+\frac{1}{2} \sigma^{2} \frac{\partial^{2} \psi}{\partial \xi^{2}} .
$$

We find that the leading term $\psi_{0}$ is again given by

$$
\psi_{0}(\tau, \xi)=a(\tau)+b(\tau) \xi
$$

therefore, no boundary layer effect or corner layer effect occurs near $r=0$ since there is no exponential decay. In this case, (3.3), (3.4), and (3.5) are a regular perturbation problem.

In order to derive a uniformly valid asymptotic solution for this case, we expand the solution $\phi(\tau, r)$ of (3.3), (3.4), and (3.5) in terms of interest rate $\beta$ as a perturbation series

$$
\phi(\tau, r)=\sum_{n=0}^{\infty} \phi_{n}(\tau, r) \beta^{n} .
$$

Making the substitution of (3.25) into (3.3), (3.4), and (3.5) and equating the coefficients of like power of $\beta$, we convert the original equations to the following equations:

$$
\begin{gathered}
\frac{\partial \phi_{0}}{\partial \tau}=\alpha \frac{\partial \phi_{0}}{\partial r}+\frac{1}{2} \sigma^{2} \frac{\partial^{2} \phi_{0}}{\partial r^{2}}=0 \\
\phi_{0}(\tau, r)=1, \quad r<0, \\
\phi_{0}(0, r)=0, \quad r>0
\end{gathered}
$$


and for $n \geq 1$, we have the following equations:

$$
\begin{aligned}
\frac{\partial \phi_{n}}{\partial \tau}-\alpha \frac{\partial \phi_{n}}{\partial r}-\frac{1}{2} \sigma^{2} \frac{\partial^{2} \phi_{n}}{\partial r^{2}} & =r e^{A(\tau)} \frac{\partial \phi_{n-1}}{\partial r} \\
\phi_{n}(\tau, r) & =0, \quad r<0, \\
\phi_{n}(0, r) & =0, \quad r>0 .
\end{aligned}
$$

The solution $\phi_{0}(\tau, r)$ of (3.26) employing the Green's function representation has the form

$$
\begin{aligned}
& \phi_{0}(\tau, r) \\
&=1-(1-H(-r))[ {\left[\int_{0}^{\infty} \frac{1}{\sigma \sqrt{2 \pi \tau}} \exp \left(-\frac{(r+\alpha \tau-\xi)^{2}}{2 \sigma^{2} \tau}\right) d \xi\right.} \\
&\left.-\int_{0}^{\infty} \frac{1}{\sigma \sqrt{2 \pi \tau}} \exp \left(\frac{2 \alpha \xi}{\sigma^{2}}\right) \exp \left(-\frac{(r+\alpha \tau+\xi)^{2}}{2 \sigma^{2} \tau}\right) d \xi\right],
\end{aligned}
$$

where function $H$ is the Heaviside function defined by

$$
H(x)= \begin{cases}1, & \text { if } x>0 \\ 0, & \text { if } x<0\end{cases}
$$

We can simplify (3.28) to obtain

$$
\begin{aligned}
\phi_{0}(\tau, r)=1-\frac{1}{2}(1-H(-r))( & 1-e^{\left(-2 r \alpha / \sigma^{2}\right)}+\operatorname{erf}\left(\frac{(r+\alpha \tau)}{\sigma \sqrt{2 \tau}}\right) \\
& \left.-e^{\left(-2 r \alpha / \sigma^{2}\right)} \operatorname{erf}\left(\frac{(\alpha \tau-r)}{\sigma \sqrt{2 \tau}}\right)\right) .
\end{aligned}
$$

Solution $\phi_{n}(\tau, r)$ of (3.27) for $n \geq 1$ has the form

$$
\phi_{n}(\tau, r)=\int_{0}^{\tau} \int_{0}^{\infty} \frac{\partial \phi_{n-1}(u, \xi)}{\partial \xi} \xi e^{A(u)} G(r, \tau ; \xi, u) d \xi d u
$$

where $G(r, \tau ; \xi, u)$ is the Green's function representation of the form

$$
\begin{aligned}
G(r, \tau ; \xi, u)= & \frac{1}{\sigma \sqrt{2 \pi(\tau-u)}} \exp \left(-\frac{(r+\alpha(\tau-u)-\xi)^{2}}{2 \sigma^{2}(\tau-u)}\right) \\
& -\exp \left(\frac{2 \alpha \xi}{\sigma^{2}}\right) \frac{1}{\sigma \sqrt{2 \pi(\tau-u)}} \exp \left(-\frac{(r+\alpha(\tau-u)+\xi)^{2}}{2 \sigma^{2}(\tau-u)}\right) .
\end{aligned}
$$


In order to simplify (3.31), we let

$$
\begin{aligned}
\Psi_{n-1}(\xi) & =\frac{\partial \phi_{n-1}(u, \xi)}{\partial \xi} \xi e^{A(u)}, \\
\hat{t} & =\tau-u .
\end{aligned}
$$

Then, for $n \geq 1$, the integral

$$
\begin{aligned}
I= & \int_{0}^{\infty} \frac{\partial \phi_{n-1}(\xi)}{\partial \xi} \xi e^{A(u)} G(r, \tau ; \xi, u) d \xi \\
= & \int_{0}^{\infty} \Psi_{n-1}(u, \xi)\left[\frac{1}{\sigma \sqrt{2 \pi \hat{t}}} \exp \left(-\frac{(r+\alpha \hat{t}-\xi)^{2}}{2 \sigma^{2} \hat{t}}\right)\right. \\
& \left.\quad-\exp \left(\frac{2 \alpha \xi}{\sigma^{2}}\right) \frac{1}{\sigma \sqrt{2 \pi \hat{t}}} \exp \left(-\frac{(r+\alpha \hat{t}+\xi)^{2}}{2 \sigma^{2} \hat{t}}\right)\right] d \xi \\
= & \int_{-\infty}^{r} \frac{\Psi_{n-1}(r-s)}{\sigma \sqrt{2 \pi \hat{t}}} \exp \left(-\frac{(s+\alpha \hat{t})^{2}}{2 \sigma^{2} \hat{t}}\right) d s \\
& -\exp \left(-\frac{\alpha r}{\sigma^{2}}\right) \int_{r}^{\infty} \frac{\Psi_{n-1}(s-r)}{\sigma \sqrt{2 \pi \hat{t}}} \exp \left(-\frac{(s-\alpha \hat{t})^{2}}{2 \sigma^{2} \hat{t}}\right) d s .
\end{aligned}
$$

Let $\eta=(s+\alpha \hat{t}) / 2 \sqrt{\hat{t}}$, then we have

$$
\begin{aligned}
I & =\int_{0}^{\infty} \frac{\partial \phi_{n-1}(u, \xi)}{\partial \xi} \xi e^{A(u)} G(r, \tau ; \xi, u) d \xi \\
& =I_{1}(r, \hat{t})-I_{2}(r, \hat{t}) \exp \left(-\frac{\alpha r}{\sigma^{2}}\right),
\end{aligned}
$$

where

$$
\begin{aligned}
& I_{1}(r, \hat{t})=\sqrt{\frac{2}{\pi \sigma^{2}}} \int_{-\infty}^{(r+\alpha \hat{t}) / 2 \sqrt{\hat{t}}} \Psi_{n-1}(r+\alpha \hat{t}-2 \eta \sqrt{\hat{t}}) \exp \left(-\frac{\eta^{2}}{\sigma^{2} / 2}\right) d \eta, \\
& I_{2}(r, \hat{t})=\sqrt{\frac{2}{\pi \sigma^{2}}} \int_{(r-\alpha \hat{t}) / 2 \sqrt{\hat{t}}}^{\infty} \Psi_{n-1}(\alpha \hat{t}+2 \eta \sqrt{\hat{t}}-r) \exp \left(-\frac{\eta^{2}}{\sigma^{2} / 2}\right) d \eta .
\end{aligned}
$$

For $n \geq 1$, we have the solution $\phi_{n}(\tau, r)$ of (3.31)

$$
\phi_{n}(\tau, r)=\int_{0}^{\tau}\left[I_{1}(r, \hat{t})-I_{2}(r, \hat{t}) \exp \left(-\frac{\alpha r}{\sigma^{2}}\right)\right] d \hat{t}
$$


where $I_{1}(r, \hat{t})$ and $I_{2}(r, \hat{t})$ are defined in (3.36), $\hat{t}=T-t=u$, and

$$
\Psi_{n-1}(\xi)=\frac{\partial \phi_{n-1}(u, \xi)}{\partial \xi} \xi e^{A(u)}
$$

Therefore, we have the following theorem.

THEOREM 3.3. For $\alpha \leq 0$, the asymptotic solution $\phi_{n}(t, r)$ of (3.1) and (3.2) has the following form:

$$
\phi(t, r)=\phi_{0}(t, r)+\sum_{n=1}^{\infty} \phi_{n}(t, r) \beta^{n}
$$

with

$$
\begin{array}{r}
\phi_{0}(t, r)=1-\frac{1}{2}(1-H(-r))\left(1-e^{\left(-2 r \alpha / \sigma^{2}\right)}+\operatorname{erf}\left(\frac{(r+\alpha(T-t))}{\sigma \sqrt{2(T-t)}}\right)\right. \\
\left.-e^{\left(-2 r \alpha / \sigma^{2}\right)} \operatorname{erf}\left(\frac{(\alpha(T-t)-r)}{\sigma \sqrt{2(T-t)}}\right)\right)
\end{array}
$$

and $\phi_{n}(t, r)$ for $n \geq 1$, as defined in (3.37), is uniformly valid in the domain of $r$ and $t$.

For the case $\sigma$ is small, since the integrals $I_{1}(r, \hat{t})$ and $I_{2}(r, \hat{t})$ are of the same forms as in (2.11) and (2.15) in Theorem 2.5, respectively, we can apply Theorem 2.5 to (3.35) repeatedly and obtain

$$
\begin{aligned}
& I=\int_{0}^{\infty} \frac{\partial \phi_{n-1}(u, \xi)}{\partial \xi} \xi e^{A(u)} G(r, \tau ; \xi, u) d \xi \\
& =\sum_{k=0}^{m-1}\left[\sigma ^ { 2 k } \left\{\frac{1}{4^{k}} \Omega_{1}^{(k)}(0 ; r, \hat{t})-\frac{1}{2^{2 k+1}} \Omega_{1}^{(k)}\left(\frac{r+\alpha \hat{t}}{2 \sqrt{\hat{t}}}\right) \operatorname{erfc}\left(\frac{r+\alpha \hat{t}}{\sigma \sqrt{2 \hat{t}}}\right)\right.\right. \\
& \left.\quad-\frac{1}{2^{2 k+1}} \Omega_{2}^{(k)}\left(\frac{r-\alpha \hat{t}}{2 \sqrt{\hat{t}}}\right) e^{\left(-2 \alpha r / \sigma^{2}\right)} \operatorname{erfc}\left(\frac{r-\alpha \hat{t}}{\sigma \sqrt{2 \hat{t}}}\right)\right\} \\
& +\sigma^{2 k+1}\left\{\sqrt { \frac { \hat { t } } { 2 } } \left(\frac{\Omega_{1}^{(k)}(0 ; r, \hat{t})-\Omega_{1}^{(k)}((r+\alpha \hat{t}) / 2 \sqrt{\hat{t}} ; r, \hat{t})}{2^{k}(r+\alpha \hat{t})} \operatorname{ierfc}\left(\frac{r+\alpha \hat{t}}{\sigma \sqrt{2 \hat{t}}}\right)\right.\right. \\
& +\Omega_{2}^{(k)}(0 ; r, \hat{t})-\Omega_{2}^{(k)}((r-\alpha \hat{t}) / 2 \sqrt{\hat{t}} ; r, \hat{t}) \\
& 2^{k}(r-\alpha \hat{t})
\end{aligned}
$$


where

$$
\begin{aligned}
\Omega_{1}^{(k)}(0 ; r, \hat{t}) & =\lim _{s \rightarrow 0} \Omega_{1}^{(k)}(s ; r, \hat{t}), \\
\Omega_{2}^{(k)}(0 ; r, \hat{t}) & =\lim _{s \rightarrow 0} \Omega_{2}^{(k)}(s ; r, \hat{t}), \\
\Omega_{1}^{(k)}(s ; r, \hat{t}) & = \begin{cases}\Psi_{n-1}(r+\alpha \hat{t}-2 s \sqrt{\hat{t}}), & \text { if } k=0, \\
\frac{d}{d s}\left[\frac{1}{s}\left\{\Omega_{1}^{(k-1)}(s ; r, \hat{t})-\Omega_{1}^{(k-1)}(0 ; r, \hat{t})\right\}\right], & \text { if } k \geq 1,\end{cases} \\
\Omega_{2}^{(k)}(s ; r, \hat{t}) & = \begin{cases}\Psi_{n-1}(-r+\alpha \hat{t}+2 s \sqrt{\hat{t}}), & \text { if } k=0, \\
\frac{d}{d s}\left[\frac{1}{s}\left\{\Omega_{2}^{(k-1)}(s ; r, \hat{t})-\Omega_{2}^{(k-1)}(0 ; r, \hat{t})\right\}\right], & \text { if } k \geq 1,\end{cases} \\
I_{1}^{(m)}(r, \hat{t}) & =\sqrt{\frac{2}{\pi \sigma^{2}} \int_{-\infty}^{(r+\alpha t) / 2 \sqrt{\hat{t}}} \Omega_{1}^{(m)}(\eta ; r, \hat{t}) \exp \left(-\frac{\eta^{2}}{\sigma^{2} / 2}\right) d \eta,} \\
I_{2}^{(m)}(r, \hat{t}) & =\sqrt{\frac{2}{\pi \sigma^{2}} \int_{(r+\alpha t) / 2 \sqrt{\hat{t}}}^{\infty} \Omega_{2}^{(m)}(\eta ; r, \hat{t}) \exp \left(-\frac{\eta^{2}}{\sigma^{2} / 2}\right) d \eta .}
\end{aligned}
$$

Therefore, together with (3.30), we have the solution $\phi_{n}(\tau, r)$ of (3.27) in terms of an asymptotic uniform expansion in $\sigma$

$$
\begin{aligned}
& \phi_{n}(\tau, r) \\
& =\sum_{k=0}^{m-1}\left[\sigma ^ { 2 k } \left\{\frac{1}{4^{k}} \int_{0}^{\tau} \Omega_{1}^{(k)}(0 ; r, \hat{t}) d \hat{t}\right.\right. \\
& -\frac{1}{2^{2 k+1}} \int_{0}^{\tau}\left[\Omega_{1}^{(k)}\left(\frac{r+\alpha \hat{t}}{2 \sqrt{\hat{t}}}\right) \operatorname{erfc}\left(\frac{r+\alpha \hat{t}}{\sigma \sqrt{2 \hat{t}}}\right)\right. \\
& \left.\left.+\Omega_{2}^{(k)}\left(\frac{r-\alpha \hat{t}}{2 \sqrt{\hat{t}}}\right) e^{\left(-2 \alpha r / \sigma^{2}\right)} \operatorname{erfc}\left(\frac{r-\alpha \hat{t}}{\sigma \sqrt{2 \hat{t}}}\right)\right] d \hat{t}\right\} \\
& +\sigma^{2 k+1} \int_{0}^{\tau}\left\{\sqrt { \frac { \hat { t } } { 2 } } \left[\frac{\Omega_{1}^{(k)}(0 ; r, \hat{t})-\Omega_{1}^{(k)}((r+\alpha \hat{t}) / 2 \sqrt{\hat{t}} ; r, \hat{t})}{2^{k}(r+\alpha \hat{t})} \operatorname{ierfc}\left(\frac{r+\alpha \hat{t}}{\sigma \sqrt{2 \hat{t}}}\right)\right.\right. \\
& +\frac{\Omega_{2}^{(k)}(0 ; r, \hat{t})-\Omega_{2}^{(k)}((r-\alpha \hat{t}) / 2 \sqrt{\hat{t}} ; r, \hat{t})}{2^{k}(r-\alpha \hat{t})} \\
& \left.\left.\left.\times e^{\left(-2 \alpha r / \sigma^{2}\right)} \operatorname{ierfc}\left(\frac{r-\alpha \hat{t}}{\sigma \sqrt{2 \hat{t}}}\right)\right]\right\} d \hat{t}\right] \\
& +\sigma^{2 m} R^{(m)}(r, \hat{t} ; \sigma),
\end{aligned}
$$

with erfc and ierfc as defined in (2.16) and (2.17); the remainder term

$$
R^{(m)}(r, \hat{t} ; \sigma)=\frac{1}{4^{m}} \int_{0}^{\tau}\left\{I_{1}^{(m)}(r, \hat{t})-I_{2}^{(m)}(r, \hat{t})\right\} d \hat{t}
$$

where $I_{1}^{(m)}(r, \hat{t})$ and $I_{2}^{(m)}(r, \hat{t})$ are as defined in (3.46) and (3.47), respectively. 
Therefore, we have the following theorem.

THEOREM 3.4. For $\alpha \leq 0$ and $\sigma \ll 1$, the solution $\phi_{n}(t, r)$ of (3.3), (3.4), and (3.5) is

$$
\phi(t, r)=\phi_{0}(t, r)+\sum_{n=1}^{\infty} \phi_{n}(t, r) \beta^{n}
$$

with $\phi_{0}(t, r)$ as given in (3.40), and $\phi_{n}(t, r)$ for $n \geq 1$ as defined in (3.47). The expansion (3.50) is uniformly valid in the whole domain for all $\beta, \sigma>0$ and

$$
\begin{aligned}
& \phi_{n}(t, r) \\
& \sim \sum_{k=0}^{m-1}\left[\frac{\sigma^{2 k}}{4^{k}} \int_{0}^{T-t} \Omega_{1}^{(k)}(0 ; r, \hat{t}) d \hat{t}\right. \\
& -\frac{\sigma^{2 k+2}}{2^{2 k+1} \sqrt{\pi}} \int_{0}^{\tau} \exp \left(-\frac{(r+\alpha \hat{t})^{2}}{2 \sigma^{2} \hat{t}}\right)\left[\Omega_{1}^{(k)}\left(\frac{r+\alpha \hat{t}}{2 \sqrt{\hat{t}}} ; r, \hat{t}\right)\left(\frac{\hat{t}}{r+\alpha \hat{t}}\right)\right. \\
& \left.+\Omega_{2}^{(k)}\left(\frac{r-\alpha \hat{t}}{2 \sqrt{\hat{t}}} ; r, \hat{t}\right)\left(\frac{\hat{t}}{r-\alpha \hat{t}}\right)\right] d \hat{t} \\
& +\sigma^{2 k+3} \int_{0}^{\tau}\left\{\frac{\hat{t}^{3 / 2}}{\sqrt{2}} \exp \left(-\frac{(r+\alpha \hat{t})^{2}}{2 \sigma^{2} \hat{t}}\right)\right. \\
& \times\left[\frac{\Omega_{1}^{(k)}(0 ; r, \hat{t})-\Omega_{1}^{(k)}((r+\alpha \hat{t}) / 2 \sqrt{\hat{t}} ; r, \hat{t})}{2^{k}(r+\alpha \hat{t})^{2}}\right. \\
& \left.\left.\left.+\frac{\Omega_{2}^{(k)}(0 ; r, \hat{t})-\Omega_{2}^{(k)}((r-\alpha \hat{t}) / 2 \sqrt{\hat{t}} ; r, \hat{t})}{2^{k}(r-\alpha \hat{t})^{2}}\right]\right\} d \hat{t}\right] \\
& +\sigma^{2 m} R^{(m)}(r, \hat{t} ; \sigma)
\end{aligned}
$$

We now plot some results to illustrate the asymptotic behavior of the solutions (1.1), (1.2), and (1.3) with different values of the interest rate $\beta$ and the diffusion coefficient $\sigma$.

When $\alpha=1, T=1$, and interest rate $\beta=0$, for (I) $\sigma=0.0005$ and (II) $\sigma=1$, the asymptotic behavior of the conditional ruin probability are illustrated by the following figures.

We notice in Figures 3.1 and 3.2 that the conditional probability of ruin $\phi(t, r)$ reaches zero much faster in Case (I) (reserve $r \approx 10^{-6}$ ) than in Case (II) (reserve $r \approx 1.6$ ).

For other fixed parameters, Figure 3.3 shows the behavior of the conditional ruin probability as time decreases. Figure 3.4 shows the behavior of the ruin probability as the reserve level decreases. Figure 3.5 shows that the conditional ruin probability decreases as the interest rate increases.

Figures 3.6 and 3.7 show that the conditional ruin probability decreases as the variance $\sigma$ decreases. 


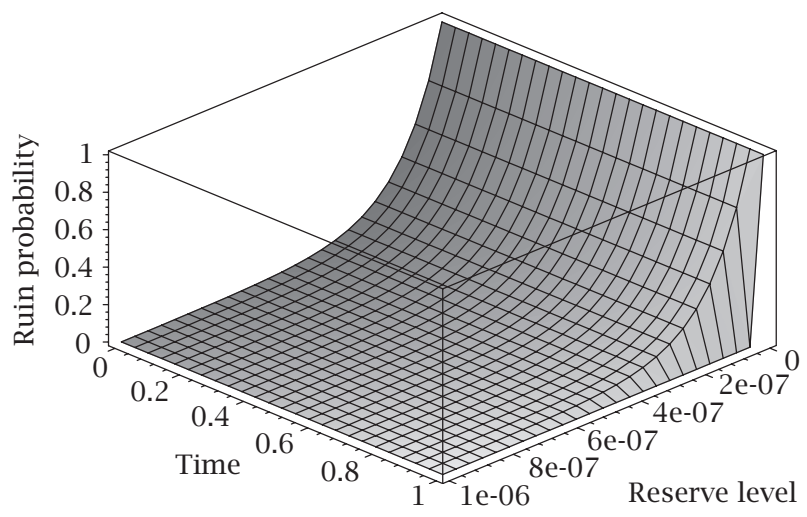

FIGURE 3.1. Probability of ruin for case (I).

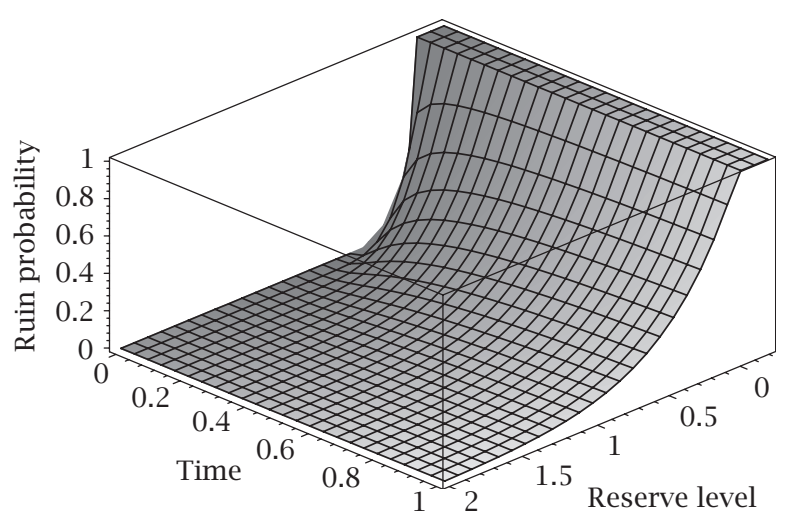

FIGURE 3.2. Probability of ruin for case (II).

4. Conclusion and remarks. In study of diffusion models for risk reserves, a special class of initial boundary value problem of linear diffusion equations (1.1), (1.2), and (1.3) is obtained. Using asymptotic analysis and perturbation theory, we provide the asymptotic behavior of the conditional probability of ruin over finite time for different cases. Our results show that when the difference $\alpha$ of the aggregate rate $\pi(t, r)$ at which premiums are cashed that time and the average aggregated claim rate $\mu(t)$ at time $t$ is greater than zero, there are boundary layer and parabolic corner layer occur if volatility coefficient $\sigma$ is small, or it is order of the small interest rate. In this case, inflation has little effect on the asymptotic behavior of the ruin probability $\phi(t, r)$. However, there is no boundary layer or corner layer behavior when $\alpha \leq 0$, then the asymptotic solutions of (1.1), (1.2), and (1.3) can be obtained in terms of 


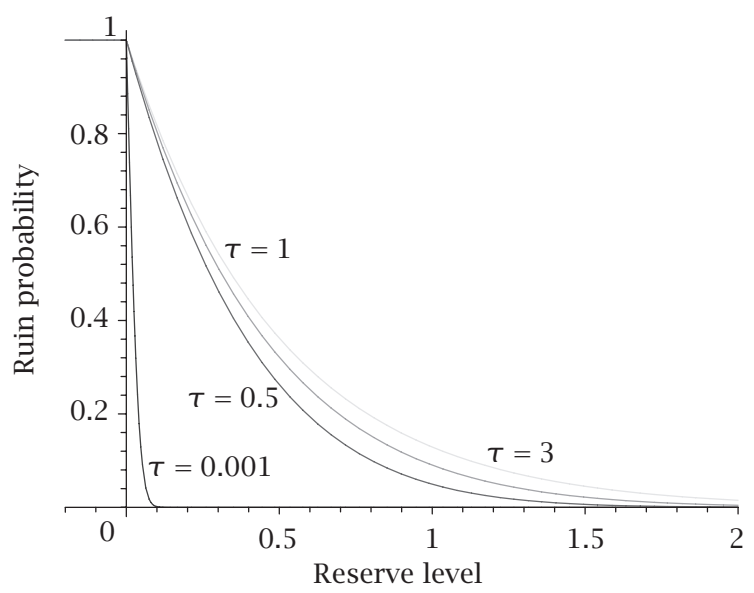

FIGURE 3.3

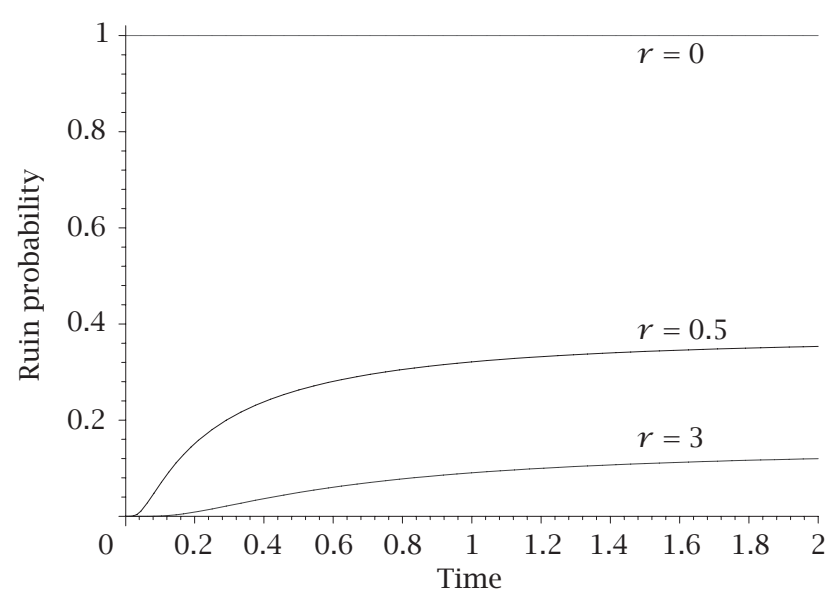

FIGURE 3.4

interest rate $\beta$, which is uniformly valid in the given domain. The result also provides us with some insight of the asymptotic behavior of the conditional ruin probability for a more general model (2.8) where $\pi(t, r)-\mu(t)=r g(t)$. The similar techniques can be applied to obtain the asymptotic behavior of the solutions by converting (2.8) into the heat equation via a transformation in both independent variables $t$ and $r$.

In addition, our approach to the reserve process which is described by a single stochastic differential equation in the above sections can be extended to a vector form of stochastic differential equation. In fact, a company may hold different portfolios. If we assume that there are $n$ portfolios, then the risk 


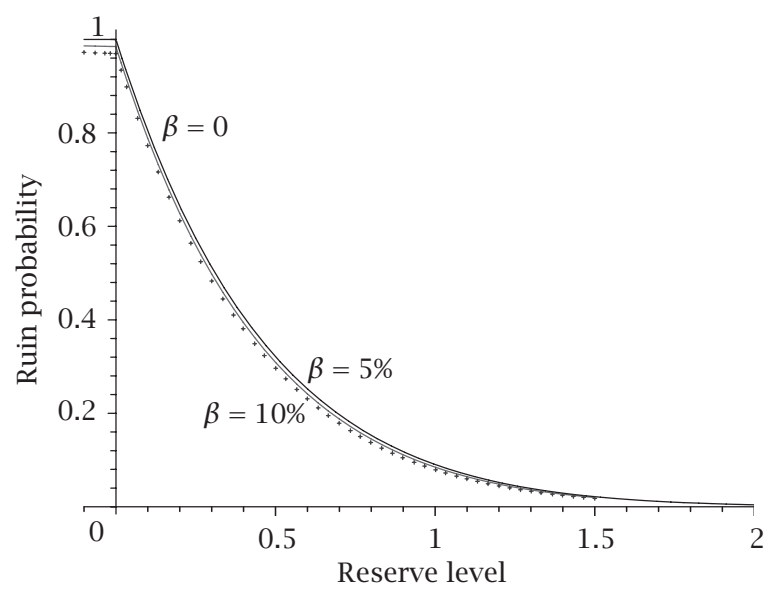

FIGURE 3.5

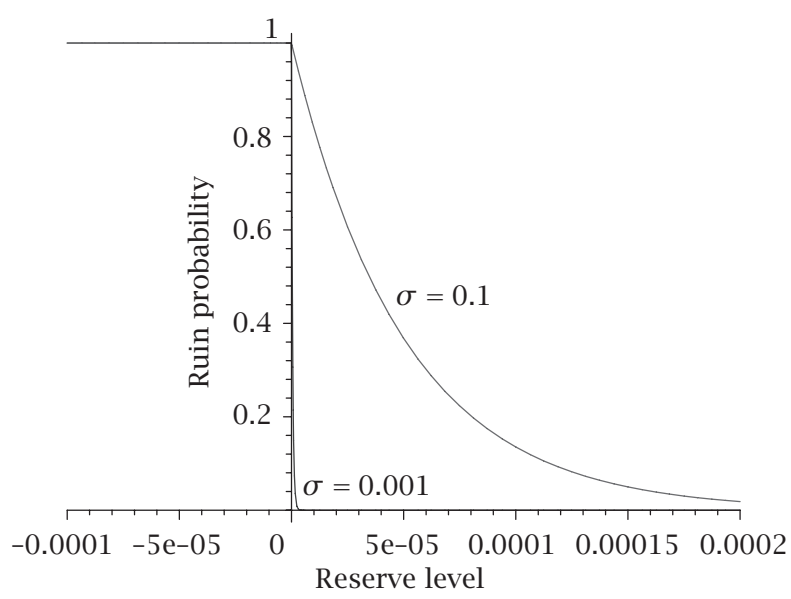

FIGURE 3.6

asset and risk-free asset will follow the different dynamics, and the governing equations of probability of ruin are described by (4.1). In this case, the vector reserve processes can be described by a vector form of (2.7). Similar techniques can provide a family of parabolic partial differential equation

$$
\begin{gathered}
\frac{\partial \phi}{\partial t}+\sum_{i=1}^{n} \frac{\partial \phi}{\partial r_{i}} a_{i}(t, r)+\frac{1}{2} \sigma^{2} \sum_{i=1}^{n} \sum_{j=1}^{m}\left[\frac{\partial^{2} \phi}{\partial r_{i} r_{j}}(t, r)+\frac{\partial \phi}{\partial r_{i}} \frac{\partial f}{\partial r_{j}}(t, r)\right] b_{i j}(t, r)=0, \\
\phi(t, r)=1, \quad f(r)<0, \quad \phi(T, r)=0, \quad f(r)>0,
\end{gathered}
$$




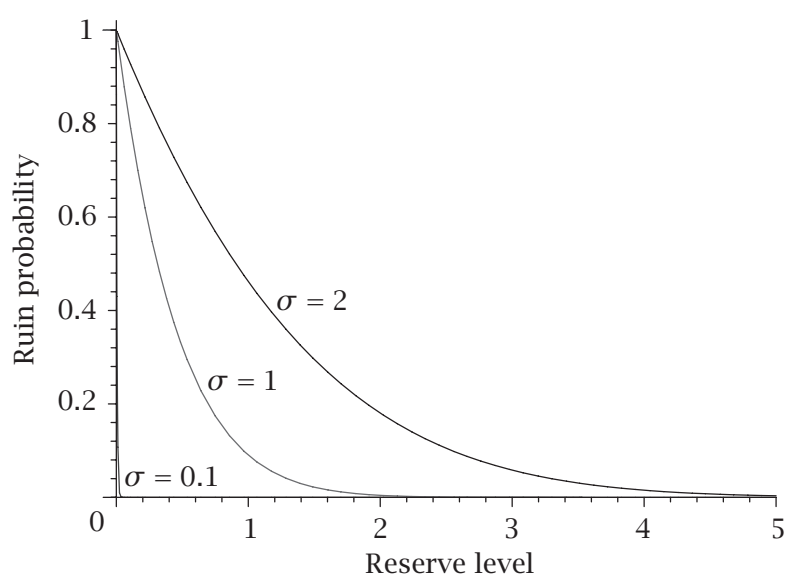

FIGURE 3.7

where $f: \mathbb{R}^{n} \rightarrow \mathbb{R}$ is a $C^{2}$ function and the probability of ruin $\phi(t, r)$ of the total reserves

$$
\phi(t, r)=P\left(\inf _{t \leq s \leq T} f(R(s))<0 \mid f(R(t))=f(r)\right) .
$$

The study of such equations will be discussed in somewhere else.

\section{REFERENCES}

[1] M. Abramowitz and I. A. Stegun, Handbook of Mathematical Functions with Formulas, Graphs, and Mathematical Tables, National Bureau of Standards Applied Mathematics Series, vol. 55, U.S. Government Printing Office, Washington, DC, 1964.

[2] R. J. Elliott, Stochastic Calculus and Applications, Applications of Mathematics, vol. 18, Springer-Verlag, Berlin, 1982.

[3] W. H. Fleming and H. M. Soner, Controlled Markov Processes and Viscosity Solutions, Applications of Mathematics, vol. 25, Springer-Verlag, New York, 1993.

[4] M. Freidlin, Functional Integration and Partial Differential Equations, Annals of Mathematics Studies, vol. 109, Princeton University Press, New Jersey, 1985.

[5] J. Garrido, Diffusion premiums for claim severities subject to inflation, Insurance Math. Econom. 7 (1988), no. 2, 123-129.

[6] __ Stochastic differential equations for compounded risk reserves, Insurance Math. Econom. 8 (1989), no. 3, 165-173.

[7] J. Grandell, Aspects of Risk Theory, Springer Series in Statistics: Probability and Its Applications, Springer-Verlag, New York, 1991.

[8] J. M. Harrison, Ruin problems with compounding assets, Stochastic Processes Appl. 5 (1977), no. 1, 67-79.

[9] D. L. Iglehart, Diffusion approximations in collective risk theory, J. Appl. Probability 6 (1969), 285-292. 
[10] C. M. Moller, Stochastic differential equations for ruin probabilities, J. Appl. Probab. 32 (1995), no. 1, 74-89.

[11] M. Musiela and M. Rutkowski, Martingale Methods in Financial Modelling, Applications of Mathematics, vol. 36, Springer-Verlag, Berlin, 1997.

[12] S.-D. Shih, A novel uniform expansion for a singularly perturbed parabolic problem with corner singularity, Methods Appl. Anal. 3 (1996), no. 2, 203-227.

[13] G. E. Willmot and H. Yang, A partial differential equations satisfied by the conditional ruin probability, unpublished manuscript, 1995.

[14] V. R. Young and T. Zariphopoulou, Computation of distorted probabilities for diffusion processes via stochastic control methods, Insurance Math. Econom. 27 (2000), no. 1, 1-18.

S. Shao: Department of Mathematics, Cleveland State University, Cleveland, $\mathrm{OH}$ 44115, USA

E-mail address: s.shao@csuohio.edu 


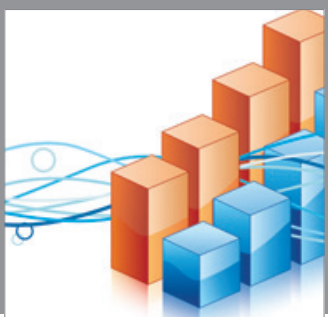

Advances in

Operations Research

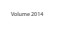

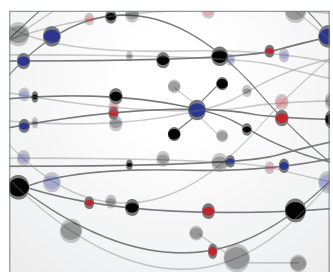

\section{The Scientific} World Journal
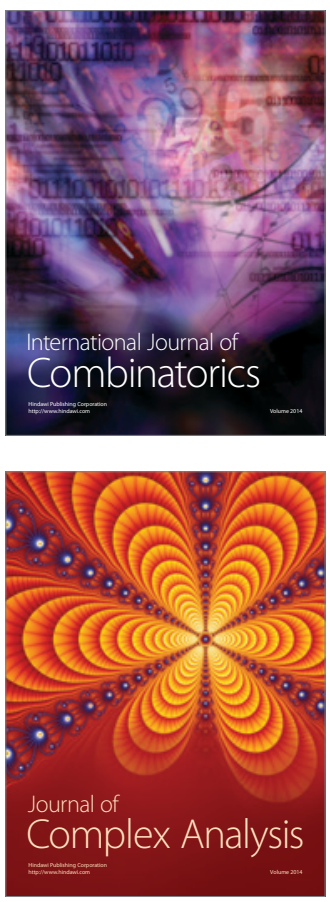

International Journal of

Mathematics and

Mathematical

Sciences
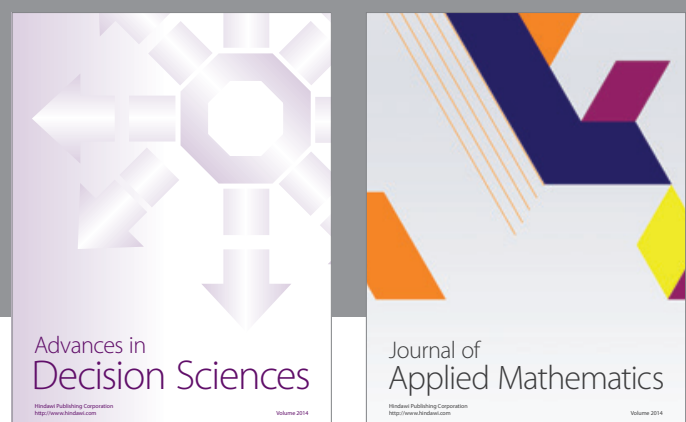

Journal of

Applied Mathematics
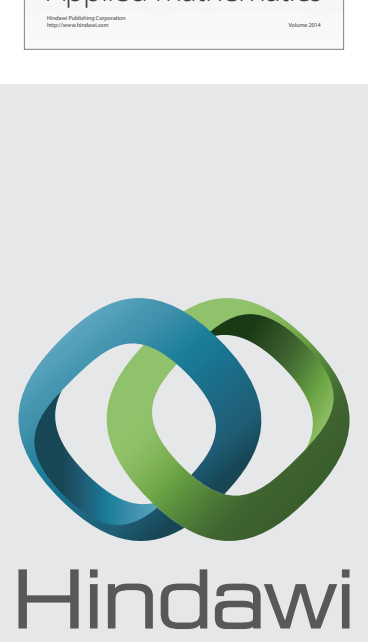

Submit your manuscripts at http://www.hindawi.com
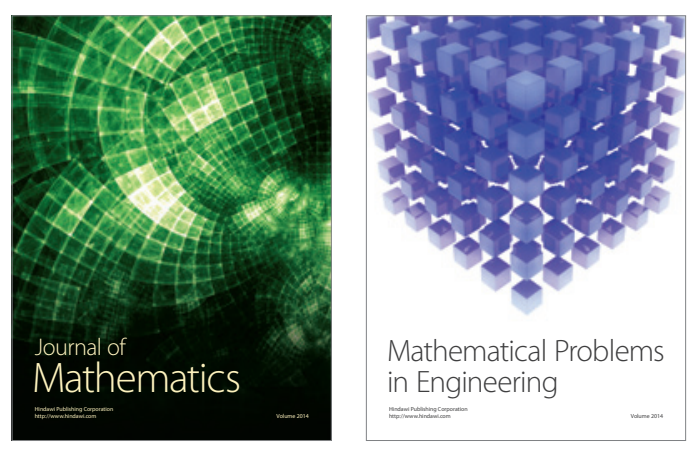

Mathematical Problems in Engineering
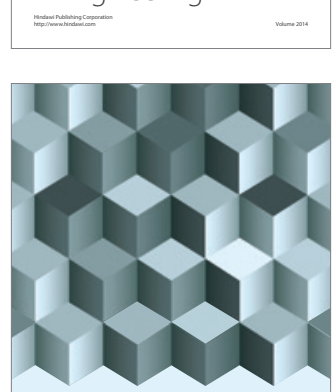

Journal of

Function Spaces
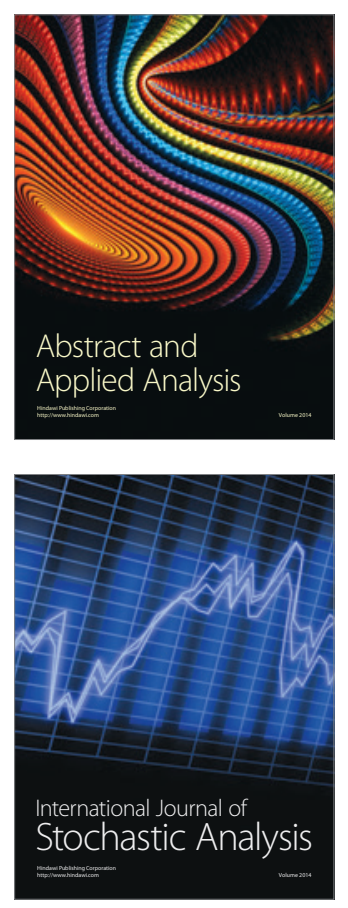

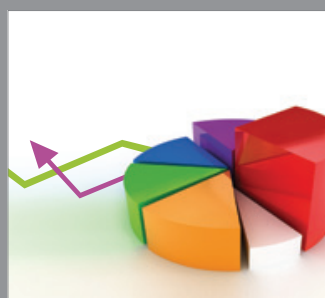

ournal of

Probability and Statistics

Promensencen
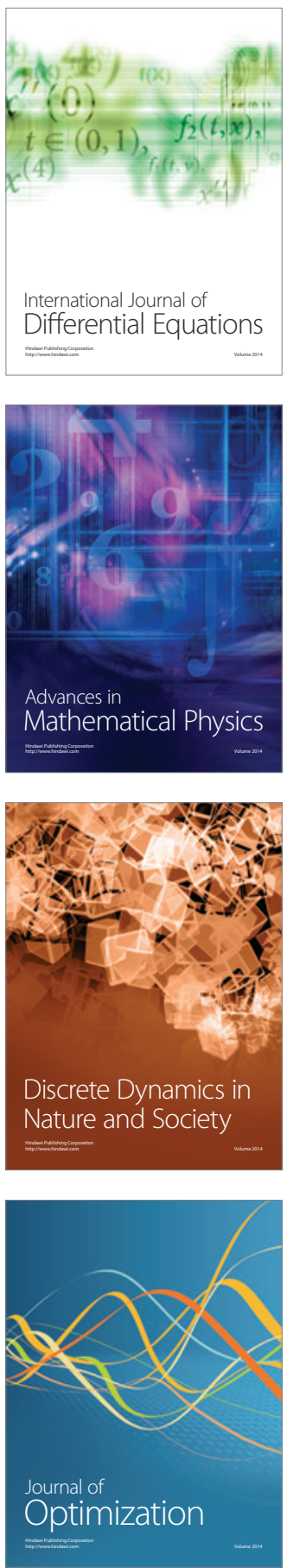